\title{
On the Capacity Limits of HVAC Duct Channel for High-Speed Internet Access
}

\author{
Ariton E. Xhafa, Member, IEEE, Ozan K. Tonguz, Member, IEEE, Ahmet G. Cepni, Student Member, IEEE, \\ Daniel D. Stancil, Fellow, IEEE, Pavel V. Nikitin, Member, IEEE, and Dagfin Brodtkorb, Member, IEEE
}

\begin{abstract}
In this paper, we report theoretical and experimental channel-capacity estimates of heating, ventilation, and air conditioning (HVAC) ducts based on multicarrier transmission that uses $M$-ary quadrature amplitude modulation and measured channel responses at the 2.4-GHz industrial, scientific, and medical band. It is shown theoretically that data rates in excess of $1 \mathrm{~Gb} / \mathrm{s}$ are possible over distances up to $500 \mathrm{~m}$ in straight ducts in which reflections have been suppressed. Our experimental results also show that even in the case of more complex HVAC duct networks (i.e., HVAC duct networks that include bends, tees, etc.) data rates over $2 \mathrm{~Gb} / \mathrm{s}$ are possible. Our estimations in this case are valid for distances of up to $22 \mathrm{~m}$, which was the maximum distance of our experimental setup. These experimental results, measured with a large-scale testbed set up at Carnegie Mellon University, Pittsburgh, PA, albeit limited in terms of transmitter-receiver separation distance, provide further evidence on the potential of HVAC systems as an attractive solution for providing communications in indoor wireless networks.
\end{abstract}

Index Terms-Heating, ventilation, and air conditioning (HVAC) systems for wireless transmission, indoor propagation, internet access, multicarrier transmission, orthogonal frequency-division multiplexing (OFDM).

\section{INTRODUCTION}

$\mathbf{H}$ IGH-SPEED Internet access in buildings (residential, office, and commercial) is one of the most important challenges that next-generation wireless networks face today. Traditional indoor wireless communication systems transmit and receive signals through the use of a network of transmitters, receivers, and antennas that are placed throughout the interior of a building [1]-[3]. However, the placement of transmitters, receivers, and antennas (communication systems) in an indoor environment is still largely a process of trial and error [4], [5]. Also, wires and cables (for example, fiber and coax) are costly to install and may require expensive upgrades when their capacity

Paper approved by R. A. Valenzuela, the Editor for Transmission Systems of the IEEE Communications Society. Manuscript received April 20, 2004; revised June 14, 2004. This work was supported by Asea Brown Boveri (ABB), Inc., Billingstad, Norway. This paper was presented in part at the IEEE International Conference on Communications, New York, NY, May 2002.

A. E. Xhafa was with the Department of Electrical and Computer Engineering, Carnegie Mellon University, Pittsburgh, PA 15213 USA. He is now with the Communications Systems Laboratory, Texas Instruments, Inc., Dallas, TX 75243 USA (e-mail: axhafa@ti.com).

O. K. Tonguz, A. G. Cepni, and D. D. Stancil are with the Department of Electrical and Computer Engineering, Carnegie Mellon University, Pittsburgh, PA 15213-3890 USA (e-mail: tonguz@ece.cmu.edu; acepni@ece.cmu.edu; stancil@cmu.edu).

P. V. Nikitin is with Intermec Technologies Corporation, Everett, WA 98203 USA (e-mail: pavel.nikitin@intermec.com).

D. Brodtkorb is with Asea Brown Boveri Corporate Research, N-1735 Billingstad, Norway (e-mail: dagfin.brodtkorb@no.abb.com).

Digital Object Identifier 10.1109/TCOMM.2004.841949 is exceeded, or when new technology requires different types of wires and cables than those already installed.

An alternative approach to providing a communication infrastructure in buildings is to recognize that every building is already equipped with a microwave distribution system: the heating, ventilation, and air conditioning (HVAC) ducts [6], [7]. These ducts are designed to carry air to and from all parts of the building, but can also function as hollow waveguides for microwave signals. While the concept and preliminary results on this approach have been reported in [6]-[8], in this paper, we quantify, for the first time, theoretical estimates of HVAC duct channel capacity based on multicarrier transmission that uses $M$-ary quadrature amplitude modulation (M-QAM) and measured channel responses at the $2.4-\mathrm{GHz}$ industrial, scientific, and medical (ISM) band.

Our results show that in "matched" 1 straight ducts, datatransmission rates in excess of $1 \mathrm{~Gb} / \mathrm{s}$ are possible over distances up to $500 \mathrm{~m}$. For more complex HVAC duct networks, one tends to think that the channel capacity will reduce drastically due to the power loss in the tee-junctions, wye-junctions, etc. However, our results show that for more complex HVAC duct networks (that contains tees and bends) with distances of up to $22 \mathrm{~m}$, data-transmission rates in excess of $2 \mathrm{~Gb} / \mathrm{s}$ are possible. The results reported in this paper show that HVAC systems can be used as an alternative technology for indoor wireless networks, which could enhance the speed of wireless internet access substantially. While from an engineering perspective, much research work remains to be done to implement this technology, from a theoretical standpoint, the capacity limits of HVAC duct channels point to the tremendous potential this technology has.

The remainder of this paper is organized as follows. In Section II, we describe briefly the experimental setup for channel measurement and preliminaries. In Section III, performance analysis of the HVAC duct channel is provided when multicarrier transmission is used as the transmission technology. Results and a discussion of their implications are presented in Section IV, while Section V concludes the paper. Auxiliary material is relegated to the appendix.

\section{Channel Measurements And Preliminaries}

The ultimate capability of the HVAC system for wireless transmission systems and networks depends on the channel characteristics of the HVAC duct channel, which is a waveguide. To determine this, we have made extensive channel-response

\footnotetext{
1One can think of "matched" ducts as radio-friendly ducts, since "matching" can, in principle, eliminate multipath reflections in HVAC ducts.
} 




Agilent E8358A

Vector Network Analyzer

Fig. 1. Experimental setup used for measurements. HVAC duct section shown is a generic representation, and in different experiments, different composite duct network configurations with different components (wyes, tees, bends, and straight ducts) were used.

measurements on various HVAC duct networks [9]-[12]. Next, we summarize the procedure used for these measurements.

Measurements were done on the second floor of Roberts Hall and National Robotics Engineering Consortium (NREC) on the Carnegie Mellon University (Pittsburgh, PA) campus. Wideband signal strength measurements were made with a system identical to the one used in [8]. Measurements of frequency and time response were done using an Agilent E8358A Vector Network Analyzer (VNA) in the $2.4-2.48 \mathrm{GHz}$ frequency band. We used cylindrical ducts of $30.5 \mathrm{~cm}$ and cylindrical pipes of $15 \mathrm{~cm}$ in diameter, both made of steel. The signal was transmitted through the duct by a monopole antenna of $3.1 \mathrm{~cm}$ in length (approximately quarter wavelength at $2.45 \mathrm{GHz}$ ) placed inside the cylindrical ducts. The receiver used the same type of antenna as the transmitter. Both antennas were connected to the VNA via coaxial cables (see Fig. 1).

At the moment, we do not have the capability of making measurements for complex HVAC duct networks that have high transmitter-receiver separation distance (e.g., greater than 25 $\mathrm{m})$. Therefore, in our analysis and results, we make use of the propagation model reported in [9]. In [9], it has been shown that the transfer function of an HVAC duct system, which is used for radio frequency (RF) communications in indoor environments, is given as

$$
H(\omega)=K_{Z} \sum_{n=1}^{N} Z_{n} e^{-\gamma_{n} L} \frac{\left(1+\Gamma e^{-2 \gamma_{n} L_{1}}\right)\left(1+\Gamma e^{-2 \gamma_{n} L_{2}}\right)}{1-\Gamma^{2} e^{-2 \gamma_{n}\left(L+L_{1}+L_{2}\right)}}
$$

where $K_{Z}$ is the coefficient that accounts for the impedance mismatch loss and depends on the impedances of the transmitter, the receiver, and the antenna; $Z_{n}$ is the complex antenna impedance due to mode $n ; \gamma_{n}=\alpha_{n}+j \beta_{n}$ is the complex propagation constant of mode $n$ ( $\alpha_{n}$ is the attenuation constant and $\beta_{n}$ is the propagation constant); $L$ is the distance between the transmitter and the receiver; and $L_{1}$ and $L_{2}$ are the distances from the antennas to the respective terminated ends with reflection coefficients equal to $\Gamma$. This propagation model deals with a straight duct network and can be used for various frequencies and various configurations of ducts (rectangular, cylindrical, etc.). Extension of this model to more complex elements of the network, i.e., tees, bends, wyes, etc., should be possible with techniques such as cascaded scattering matrices [13].

\section{ANALYSIS}

The performance of data-transmission systems is usually analyzed and measured in terms of the probability of error at a



Fig. 2. Channel frequency response of $11.7-\mathrm{m}$ long, 30.5-cm diameter straight HVAC duct.

given bit rate and signal-to-noise ratio (SNR). However, in practice, more often than not, the total transmitted power is limited. Therefore, for a practical HVAC system, it is more appropriate to calculate the attainable bit rate at a given error rate and under the restriction that the total power $P$ be limited.

Fig. 2 shows the frequency response of the HVAC duct channel for an 11.7-m long and 30.5-cm diameter straight duct. The root mean square (RMS) delay spread for this channel was measured to be $57 \mathrm{~ns}$, resulting in a coherence bandwidth $B_{c}$ of $0.35 \mathrm{MHz}$ (for $90 \%$ signal correlation). If one uses the whole bandwidth spectrum (i.e., single carrier) for data transmission, it is clear that since the transmission bandwidth is much larger than the coherence bandwidth (compare $80 \mathrm{MHz}$ with $0.35 \mathrm{MHz}$ ), intersymbol interference (ISI) will appear at the receiver side. One way to achieve ISI-free transmission is to use multicarrier transmission of the signals, where the signal bandwidth transmitted in each tone/carrier is limited by the coherence bandwidth of the channel. A more efficient use of bandwidth can be obtained with a multicarrier system if the spectra of the individual subchannels are permitted to overlap, while specific orthogonality constraints are imposed to facilitate separation of the subchannels at the receiver [14]-[17]. This technique is known as orthogonal frequency-division multiplexing (OFDM), and was first proposed by Bell Labs researchers R. W. Chang and R. A. Gibby in the 1960s [14], [15]. It has been shown that OFDM and its extensions (e.g., vector OFDM [20]) provide substantial performance improvements over single-carrier modulation [17]-[20].

In multicarrier transmission, the number of carriers $(N)$ used can be found as the minimum number of carriers needed for ISIfree transmission of the signal. For example, if the coherence bandwidth of the channel is $B_{c}$ and the available transmission band is $B_{t}$, then the number of carriers used for ISI-free signal transmission is given as

$$
N=\left\lceil\frac{B_{t}}{B_{c}}\right\rceil .
$$




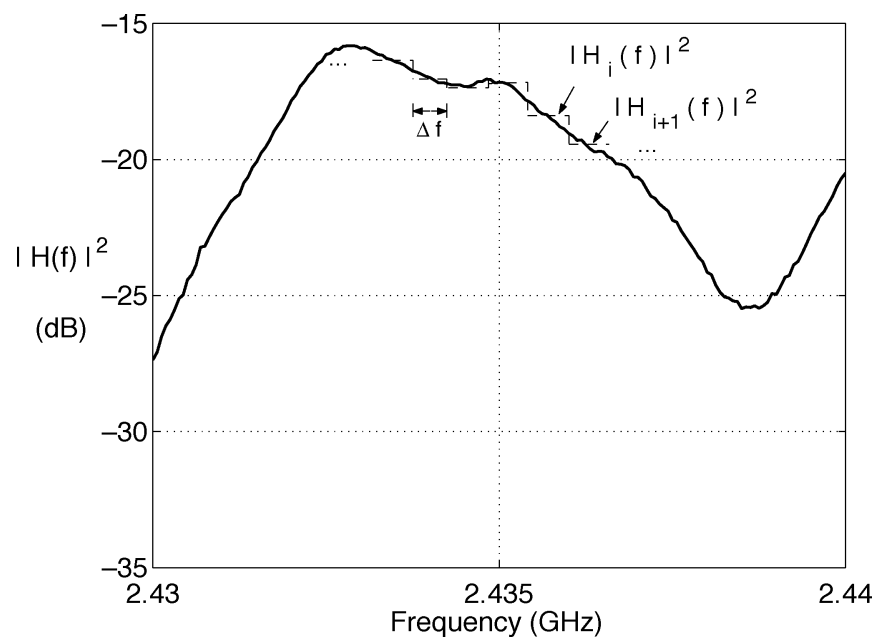

Fig. 3. Approximation of the channel-frequency response of $11.7-\mathrm{m}$ long, $30.5-\mathrm{cm}$ diameter straight HVAC duct via a staircase function for $2.43-2.44$ $\mathrm{GHz}$. Note that the figure is for illustration purposes, and that in the band $2.43-2.44 \mathrm{GHz}$, approximately 32 carriers are used.

In our analysis, we consider the use of multicarrier transmission with M-QAM ${ }^{2}$ used for each carrier. Since each carrier could transmit a different number of bits per symbol, say $n_{i}$, and assuming that ISI-free bandwidth for each carrier is $\Delta f$, then the total bit rate $R$ transmitted via the HVAC duct channel is

$$
R=\sum_{i=1}^{N} n_{i} \Delta f .
$$

In case of M-QAM and high SNR, the symbol-error probability (SEP) in the $i$ th carrier $P_{\mathrm{Se}_{i}}$ is given as [21]

$$
P_{\mathrm{se}_{i}}=4\left(1-\frac{1}{\sqrt{M_{i}}}\right) Q\left(\sqrt{\frac{3 E_{\mathrm{av}}}{\left(M_{i}-1\right) N_{0}}}\right)
$$

where $M_{i}$ is the M-QAM in the $i$ th carrier, $E_{\mathrm{av}}$ is the average energy per symbol, $Q(\cdot)$ is the error function defined as $Q(x)=(1 / \sqrt{2 \pi}) \int_{x}^{\infty} e^{-y^{2} / 2} d y$, and $N_{0}=2 N(f)$, where $N(f)$ is the double-sided power spectral density of the additive white Gaussian noise (AWGN). The SEP can also be expressed as [21]

$$
P_{\mathrm{se}_{i}}=K_{n_{i}} Q\left(\sqrt{\frac{3 E_{\mathrm{av}}}{\left(M_{i}-1\right) N_{0}}}\right)
$$

where $K_{n_{i}}$ is a constant that depends on the number of bits per symbol, such that $2 \leq K_{n_{i}} \leq 4$. In case of single-carrier transmission over a linear distorted channel of bandwidth $W$, and assuming ISI-free transmission over this channel, the number of bits per symbol (when $M$-ary modulation is used) is given as

$$
n=\log _{2} M \text {. }
$$

We also assume that M-QAM is used for single-carrier transmission. The number of bits per symbol will be lower bounded if $K_{n}=4$, and upper bounded if $K_{n}=2$. After some algebraic

\footnotetext{
${ }^{2}$ One can consider other $M$-ary modulations; however, in this paper, we study the performance of a multicarrier transmission system that uses M-QAM.
}

steps, one can show that the transmission rate over a channel with bandwidth $W$ and ISI-free transmission is given as

$$
R=W \cdot \log _{2}\left[1+\frac{\frac{3 P}{\left(N_{0} W\right)}}{\left[Q^{-1}\left\{\frac{P_{\mathrm{se}}}{K_{n}}\right\}\right]^{2}}\right]
$$

where $P$ is the transmitted signal power. In case of multicarrier transmission, approximating the channel-transfer function $H(f)$ by a staircase function (see Fig. 3) and summing over all tones/carriers, one finds that the total bit rate is given as [18], [19]

$$
R=\sum_{i=0}^{N-1} \Delta f \cdot \log _{2}\left[1+\frac{\frac{3 k_{i} P}{\left(N_{0} \Delta f\right)\left|H_{i}(f)\right|^{2}}}{\left[Q^{-1}\left\{\frac{P_{\mathrm{se}_{i}}}{K_{n_{i}}}\right\}\right]^{2}}\right]
$$

where $N$ is the number of carriers, and $k_{i}$ 's are such that

$$
\sum_{i=0}^{N-1} k_{i}=1, \quad k_{i}>0 .
$$

Assume that the symbol-error rate for each of the tones/carriers is the same, and equal to $P_{\text {se }}$. We also use $K_{n}$ instead of $K_{n_{i}}$; hence, $K_{n}=4$ or $K_{n}=2$ will lower bound or upper bound the transmission bit rate. Since each QAM signal requires at least one bit in each dimension, then in (8), one should have $\log _{2}[\cdot]>2$. Maximizing $R$ means that the power distribution has to be optimized over different frequency channels, given the constraint for $k_{i}$ 's. After some steps (see the Appendix for more details), one gets

$$
R_{\max }=\sum_{i=0}^{N-1} \Delta f \cdot \log _{2}\left[\frac{\nu}{\ln 2} K_{0}\left|H_{i}(f)\right|^{2}\right]
$$

where $K_{0}=3 P /\left(N_{0}\left[Q^{-1}\{\cdot\}\right]^{2}\right)$ and $\nu=-1 / \lambda$ is the Lagrangian multiplier.

Next, we present numerical results using different HVAC duct channel-frequency response measurements made in the laboratory.

\section{RESUlTS AND DISCUSSION}

In our calculations, we use the frequency response of an HVAC duct channel measured via the VNA. The measurements were taken using open-ended straight ducts. When multiple modes are present, reflections from open-ended ducts are small and the ducts can be considered to be "matched." It was previously verified in our measurements that the difference in the channel frequency response between closed-ended ducts with absorbers and open-ended ducts was insignificant [9]. To obtain the impulse response of the channel (that is used to calculate the coherence bandwidth of the channel), an inverse Fourier transform is performed on the frequency response of the channel. The coherence bandwidth of the channel is calculated for $90 \%$ signal correlation. Since the complexity of a practical multicarrier transmission system increases with the number of carriers, the minimum number of carriers for ISI-free signal transmission is used in the $2.4-2.48 \mathrm{GHz}$ ISM band, so that complexity is kept at the minimum possible level. Also, the number of carriers should be such that when the quantization 


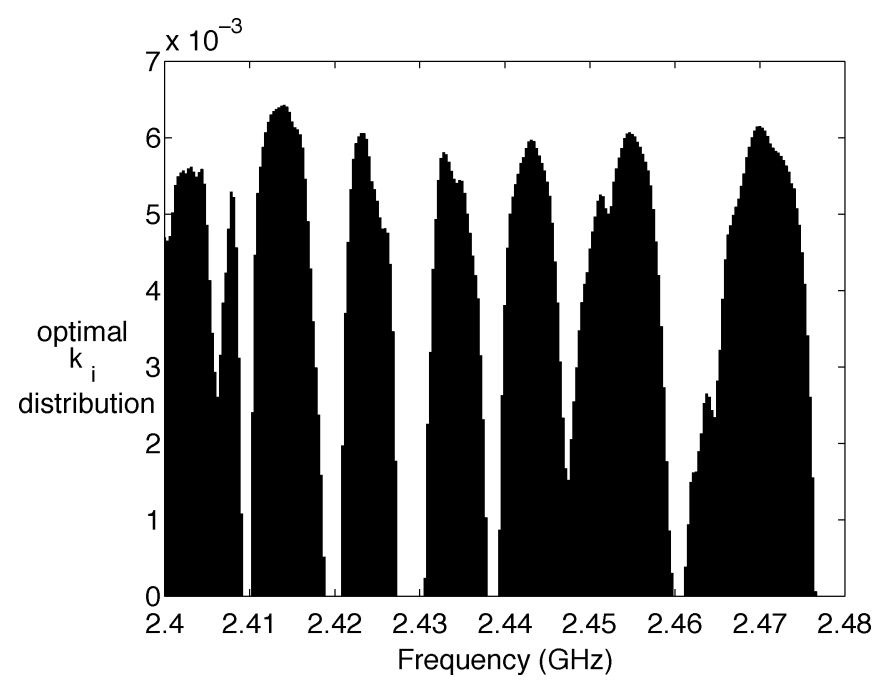

Fig. 4. Distribution of optimal values of $k_{i}$ 's for the channel frequency response of an $11.7-\mathrm{m}$ long and $30.5-\mathrm{cm}$ diameter straight HVAC duct.

of the channel frequency response is done (or equivalently, the approximation of the channel frequency response by a staircase function), the quantization error is small. In another scenario, we consider the use of a fixed number of carriers (256), where each subchannel transmission bandwidth is taken to be the minimum between the carrier spacing and the channel coherence bandwidth. The SEP was taken to be $10^{-5}$, while the value used for $K_{n}$ was four, which gives a lower bound on the HVAC duct channel capacity when one uses multicarrier transmission with M-QAM. We also assume that $P /\left(N_{0} W\right)$ is given. The values of $k_{i}$ 's associated with each of the multicarriers were found by solving (9) and (16) simultaneously.

Fig. 4 shows the distribution of the optimal values for $k_{i}$ 's (at $P /\left(N_{0} W\right)=30 \mathrm{~dB}$ ) of the channel frequency response of an 11.7-m long and 30.5-cm diameter straight HVAC duct. Comparing Fig. 4 with the channel frequency response shown in Fig. 2, one can see that the distribution of optimal values of $k_{i}$ 's follow the attenuation curve of the channel frequency response.

\section{A. HVAC Networks With Straight Ducts}

For straight ducts of $30.5-\mathrm{cm}$ diameter, we used the setup shown in Fig. 1. Making use of the channel frequency response measured from the setup shown in Fig. 1, Fig. 5 shows the HVAC duct channel capacity (normalized) versus $P /\left(N_{0} W\right)$ for measurements made for transmitter-receiver pair distances of 4.6 and $11.7 \mathrm{~m}$. For $P /\left(N_{0} W\right)$ greater than $25 \mathrm{~dB}$, a transmission data rate of more than $1 \mathrm{~Gb} / \mathrm{s}$ can be achieved for the given distances in the duct.

We repeated our experiment for straight ducts in cylindrical pipes with a diameter of $15 \mathrm{~cm}$. Note that while transmitter and receiver were located on the straight pipe, both ends of the pipe were connected to the complex network that included bends, tees, wyes, etc. Thus, the presence of multipath reflections was much higher than the case when the network consisted of simply straight "matched" ducts (as was the case in Fig. 5). Fig. 6 shows the normalized capacity versus $P /\left(N_{0} W\right)$ for different distances between the transmitter-receiver pair. Here, with $P /\left(N_{0} W\right)$ greater than $30 \mathrm{~dB}$, one could achieve $1 \mathrm{~Gb} / \mathrm{s}$

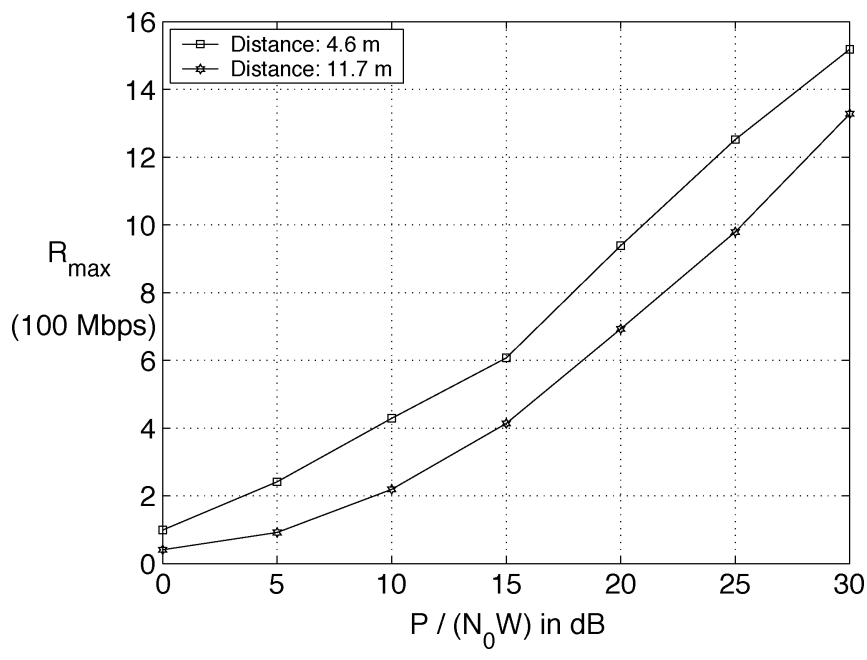

Fig. 5. Normalized capacity versus $P /\left(N_{0} W\right)$. Capacity calculations were done using experimentally measured CIRs for cylindrical ducts of $30.5-\mathrm{cm}$ diameter in the $2.4-2.48 \mathrm{GHz}$ ISM band. All 256 carriers in the $80-\mathrm{MHz}$ channel bandwidth are used in the capacity calculations.

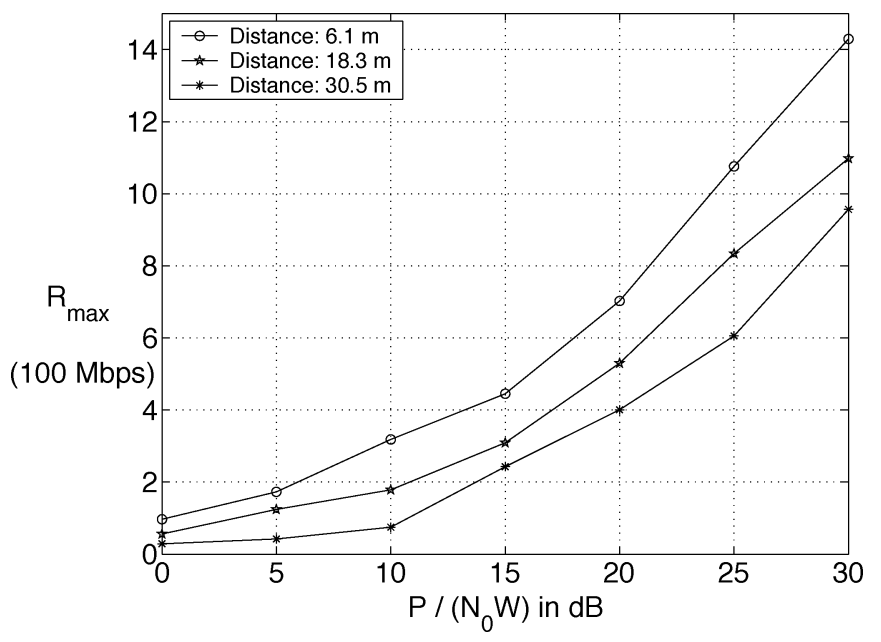

Fig. 6. Normalized capacity versus $P /\left(N_{0} W\right)$. Capacity calculations were done using experimentally measured CIRs for cylindrical pipe network of $15-\mathrm{cm}$ diameter in the 2.4-2.48 GHz ISM band. All 256 carriers in the $80-\mathrm{MHz}$ channel bandwidth are used in the capacity calculations.

or higher data-rate transmission. Observe that the channel capacity decreases as the distance between the transmitter and the receiver increases, which is the trend that one would expect. Comparing the results shown in Fig. 5 and Fig. 6, one can see that slightly higher data-transmission rates are achieved in the case of straight ducts of $30.5-\mathrm{cm}$ diameter (compare the transmission rates for distances of $4.6 \mathrm{~m}$ in Fig. 5 and $6.1 \mathrm{~m}$ in Fig. 6). However, the results shown in Fig. 5 are for straight "matched" ducts, while the results shown in Fig. 6 are for a realistic network configuration, where no effort is made to reduce the multipath reflections.

It is of interest to plot the capacity versus distance for a given transmitter power. As an example, consider a long straight open-ended ("matched") duct system. Assume a transmit power of $1 \mathrm{~W}(+30 \mathrm{dBm})$, a total receiver noise power of -90 $\mathrm{dBm}$, a probe coupling loss of $16 \mathrm{~dB}$, and a SEP of $10^{-5}$. Then, $P /\left(N_{0} W\right)=104 \mathrm{~dB}$. Capacity calculations were done using the above assumptions and channel impulse responses 


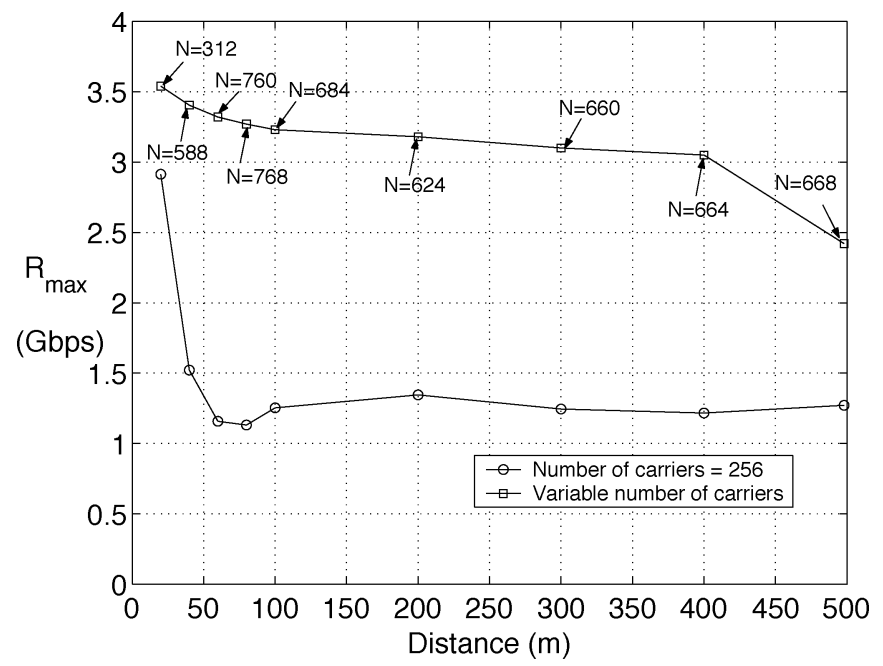

Fig. 7. Transmission rate as a function of transmitter-receiver separation distance for a transmit power of $1 \mathrm{~W}$, a receiver noise power of $-90 \mathrm{dBm}$, a probe coupling loss of $16 \mathrm{~dB}$, and a SEP of $10^{-5}$. Capacity calculations were done using CIRs extracted from our theoretical propagation model for cylindrical open-ended ducts of $30.5-\mathrm{cm}$ diameter in the $2.4-2.48 \mathrm{GHz}$ ISM band. Two cases are considered, fixed and variable numbers of carriers.

(CIRs) extracted from our theoretical propagation model [9] for different distances between the transmitter-receiver pair.

Plots of transmitted data rate as a function of distance for a fixed number of carriers (256) and a variable number of carriers are given in Fig. 7. The variable number of carriers is found as the minimum number of carriers needed for ISI-free signal transmission via an HVAC duct channel. Although the minimum number of carriers needed for ISI-free signal transmission is greater than 256 , for the fixed number of carriers case, the signal transmission bandwidth is taken to be the channel-coherence bandwidth. Note that the usable bandwidth is the channel-coherence bandwidth, even in the case of a variable number of carriers. Thus, if the coherence bandwidth of the channel is less than $B_{t} / 256$, in the case of a fixed number of carriers, a portion of $80 \mathrm{MHz}$ is not used at all, but the complexity in this case is kept at a much lower level, compared with the case with a variable number of carriers. The shape of the curves in Fig. 7 can be explained with the behavior of the RMS delay spread for different distances [11]. Since measurements were made with open-ended ducts, the RMS delay spread results from dispersion between multiple propagation modes. At short distances, this delay spread increases monotonically with distance, thus the coherence bandwidth of the channel decreases. This results in the sharp initial decline in capacity for the case of a fixed number of carriers, shown in Fig. 7 (lower curve). At longer distances, the delay spread is limited by the decay time in the ducts and the RMS delay spread (or equivalently, the coherence bandwidth) and, therefore, the capacity stabilizes for both cases.

The important observation from this figure is that, with a fixed number of carriers (i.e., $N=256$ ), bit rates in excess of $1 \mathrm{~Gb} / \mathrm{s}$ should be possible over distances up to $500 \mathrm{~m}$ in straight ducts.

However, the HVAC network in buildings does not consist of just straight ducts, but also tees, bends, wyes, etc. Thus, one has to estimate the capacity of complex HVAC duct networks. In the next section, we investigate the capacity of complex HVAC duct networks.

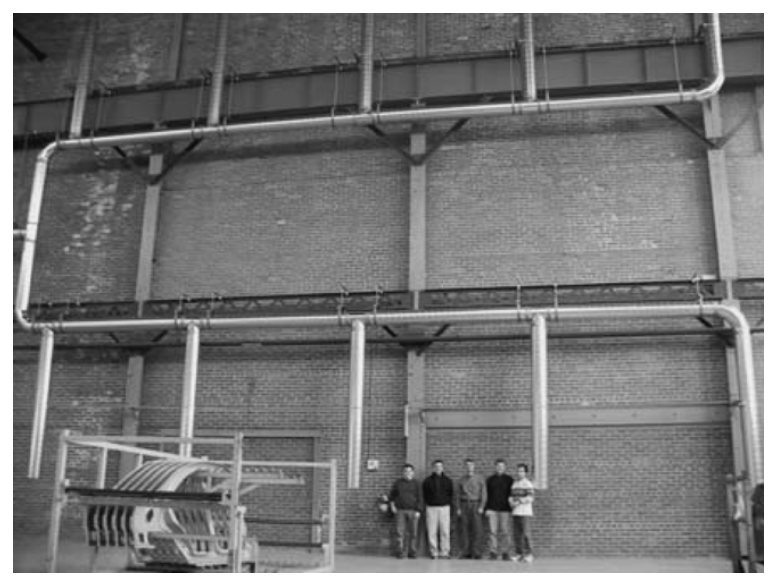

Fig. 8. Experimental setup at NREC.

\section{B. HVAC Networks With Tees and Bends}

To estimate the capacity of HVAC channels for realistic scenarios, the duct network shown in Fig. 8 (a layout is also shown in Fig. 9) was constructed at the National Robotics Engineering Consortium (NREC) Laboratory of Carnegie Mellon University. This experimental setup is representative of what might be used in office spaces in the US and Europe.

Cylindrical ducts $0.3 \mathrm{~m}$ in diameter made of galvanized steel with conductivity $\sigma=10^{6} \mathrm{~S} / \mathrm{m}$ were used for this setup. The signal was transmitted from the access point (AP) through the duct by a monopole antenna of $3.1 \mathrm{~cm}$ length. The receiver uses the same antenna as the transmitter. Both antennas were connected to an Agilent E8358A VNA via coaxial cables (as in Fig. 9). Measurements of frequency response were made using the VNA in the $2.4-2.5 \mathrm{GHz}$ frequency band. These measurements were used to calculate the data-transmission rate capabilities of this network. Assume a transmit power of $1 \mathrm{~W}(+30 \mathrm{dBm})$, a total receiver noise power of $-90 \mathrm{dBm}$, a probe coupling loss of $16 \mathrm{~dB}$, and a SEP of $10^{-5}$. Then, $P /\left(N_{0} W\right)=104 \mathrm{~dB}$. Fig. 10 shows the data-rate transmission versus transmitter-receiver separation distance for different APs (see Fig. 9). As one can see, data transmission rates over $2 \mathrm{~Gb} / \mathrm{s}$ are possible for distances up to $22 \mathrm{~m}$, even in the case of complex HVAC duct networks that include tees and bends (in addition to straight ducts). These capacity estimates are slightly lower than those of straight HVAC duct networks that have the same distance. There are two reasons for this. The first is that the power loss in a complex HVAC duct network is higher than in the straight duct networks; and the second reason is that the RMS delay spread is also higher, since more reflections are present in the complex HVAC duct networks. At the moment, we do not have the capability of making measurements for complex HVAC duct networks that have higher transmitter-receiver separation distance; however, we believe that for practical separation distances (in today's buildings, that translates into distances of up to $100 \mathrm{~m}$ ), the data-transmission capabilities of complex HVAC duct networks will also be in the gigabits-per-second range.

Our results (see Fig. 7 and Fig. 10) suggest that HVAC technology can be used as a distribution system for wireless internet 


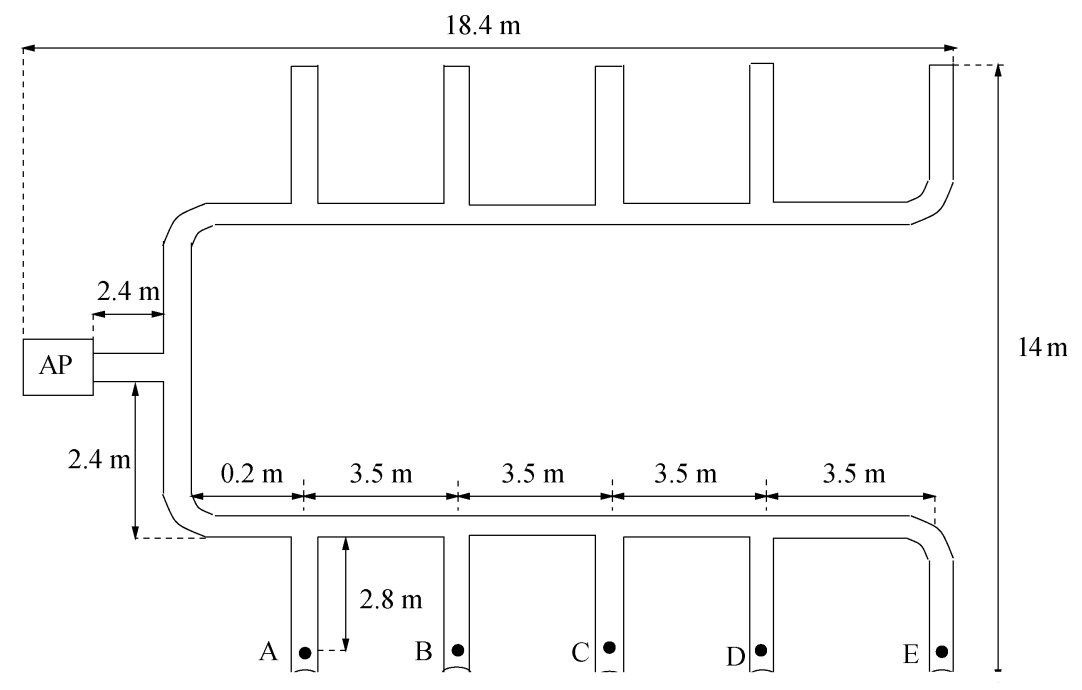

Fig. 9. Floor plan considered in the experimental setup [10].

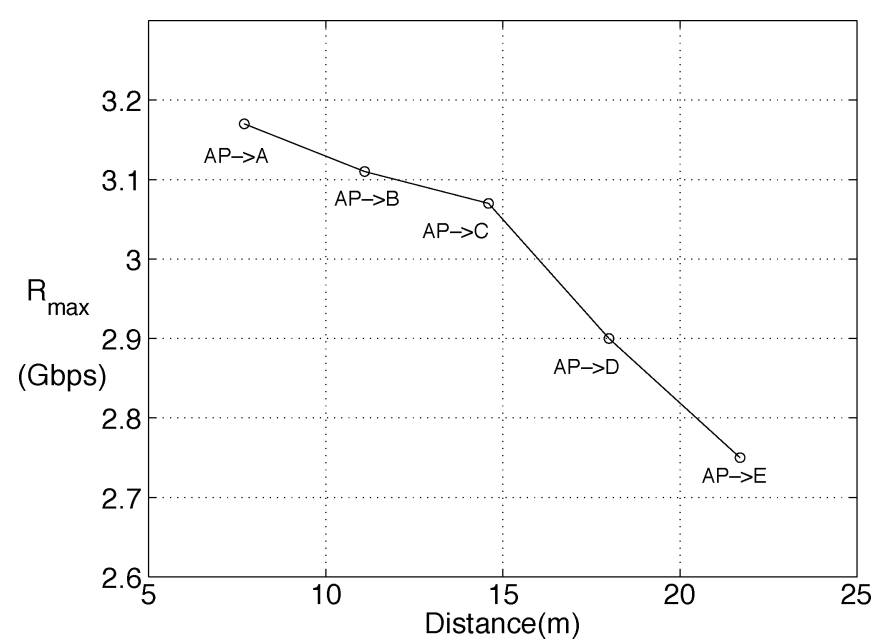

Fig. 10. Transmission rate as a function of transmitter-receiver separation distance between different APs (see Fig. 9) for a transmit power of $1 \mathrm{~W}$, a receiver noise power of $-90 \mathrm{dBm}$, a probe coupling loss of $16 \mathrm{~dB}$, and a SEP of $10^{-5}$.

access in buildings. Fig. 7 also shows that by choosing a variable number of carriers at each distance for ISI-free transmission, one can, in principle, provide data rates larger than $1 \mathrm{~Gb} / \mathrm{s}$ (see the upper curve in Fig. 7). However, to change the number of carriers for different transmitter-receiver distances over the same floor plan seems impractical and prohibitive from a complexity standpoint.

It is worth mentioning that while HVAC duct-channel capacity is an important measure for the capacity of an HVAC network, the total capacity of the network should be treated as the capacity of the compound channel, HVAC duct channel and the channel consisting of the receiving antenna pair on the duct and the user terminal. Hence, further research is needed to quantify the theoretical capacity of the compound channel.

\section{CONCLuSions}

We have quantified the theoretical channel-capacity limits of the HVAC ducts when multicarrier transmission with M-QAM is used as the transmission technology. These estimates of channel capacity based on multicarrier transmission and measured/predicted channel responses indicate that with 256 carriers in the 2.4-2.48 GHz ISM band, data rates in excess of $1 \mathrm{~Gb} / \mathrm{s}$ are possible over distances up to $500 \mathrm{~m}$ for HVAC straight ducts. For more realistic scenarios, our estimates show that data rates in excess of $2 \mathrm{~Gb} / \mathrm{s}$ are possible over distances of up to $22 \mathrm{~m}$. It is clear that the theoretical capacity estimates for HVAC channels reported in this paper suggest a new distribution technology for indoor wireless networks which could provide cheaper and faster wireless internet access to indoor environments. Thus, these theoretical capacity estimates provide further evidence on the potential of HVAC systems as an attractive and viable option for broadband wireless communications in new systems, as well as legacy systems.

\section{APPENDIX}

\section{MAXIMUM BIT RATE OF HVAC SYSTEMS}

In this appendix, we justify the maximum bit rate given in (10).

Maximizing $R$ means that the power distribution has to be optimized over different-frequency channels, given the constraint for $k_{i}$ 's. More formally, this optimization problem can be formulated as

$$
\max . R=\sum_{i=0}^{N-1} \Delta f \cdot \log _{2}\left[1+\frac{\frac{3 k_{i} P}{\left(N_{0} \Delta f\right)\left|H_{i}(f)\right|^{2}}}{\left[Q^{-1}\left\{\frac{P_{\mathrm{se}_{i}}}{K_{n_{i}}}\right\}\right]^{2}}\right]
$$

subject to the constraint that

$$
\sum_{i=0}^{N-1} k_{i}=1, \quad k_{i}>0
$$

The solution to this convex optimization problem is called the "waterpouring" solution [18], [22], and can be found using the Lagrange multiplier technique. Let $R^{\prime}$ denote a new function that is given as

$$
R^{\prime}=R+\lambda \sum_{i=0}^{N-1} k_{i}
$$


Finding the values of $k_{i}$ for which $R$ is maximized implies that $R^{\prime}$ is minimized. Hence

$$
\frac{\partial R^{\prime}}{\partial k_{i}}=0
$$

Substituting for $R^{\prime}$ yields

$$
\frac{\partial}{\partial k_{i}}\left(\sum_{i=0}^{N-1} \Delta f \cdot \log _{2}\left[1+\frac{\frac{3 k_{i} P}{\left(N_{0} \Delta f\right)\left|H_{i}(f)\right|^{2}}}{\left[Q^{-1}\left\{\frac{P_{\mathrm{se}}}{K_{n}}\right\}\right]^{2}}\right]+\lambda \sum_{i=0}^{N-1} k_{i}\right)=0
$$

which, after some algebraic steps, yields

$$
k_{i}= \begin{cases}\frac{\nu \Delta f}{\ln (2)}-\frac{\Delta f}{K_{0}\left|H_{i}(f)\right|^{2}}, & k_{i}>0 \\ 0, & \text { otherwise }\end{cases}
$$

where $K_{0}=3 P /\left(N_{0}\left[Q^{-1}\{\cdot\}\right]^{2}\right), \sum k_{i}=1$, and $\nu=-1 / \lambda$ is the Lagrangian multiplier. The Lagrangian $\nu$ and $k_{i}$ 's are found by solving (12) and (16) simultaneously. Substituting for $k_{i}$ 's in (8), the maximum bit rate is given by

$$
R_{\max }=\sum_{i=0}^{N-1} \Delta f \cdot \log _{2}\left[\frac{\nu}{\ln 2} K_{0}\left|H_{i}(f)\right|^{2}\right]
$$

which establishes (10) of the manuscript.

\section{REFERENCES}

[1] T. S. Rappaport, Wireless Communications: Principles and Practice. Englewood Cliffs, NJ: Prentice-Hall, 1996.

[2] H. L. Bertoni, Radio Propagation for Modern Wireless Systems. Englewood Cliffs, NJ: Prentice-Hall, 2000.

[3] H. Hashemi, "The indoor radio propagation channel," Proc. IEEE, vol. 81, pp. 943-968, Jul. 1993.

[4] W. Honcarenko, H. L. Bertoni, J. Dailing, and H. D. Yee, "Mechanisms governing UHF propagation on single floors in modern office buildings," IEEE Trans. Veh. Technol., vol. 41, pp. 496-504, Jul. 1992.

[5] S. S. Saunders, Antennas and Propagation for Wireless Communication Systems. New York: Wiley, 1999.

[6] C. P. Diehl, B. E. Henty, N. Kanodia, and D. D. Stancil, "Wireless RF distribution in buildings using heating and ventilation ducts," in Proc. 8th Virginia Tech/MPRG Symp. Wireless Pers. Commun., Jun. 1998, pp. 61-70.

[7] D. D. Stancil and C. P. Diehl, "Wireless Signal Distribution in a Building HVAC System," US Patent 5,977,851, Nov. 2, 1999

[8] D. D. Stancil, O. K. Tonguz, A. Xhafa, A. Cepni, P. Nikitin, and D. Brodtkorb, "High-speed internet access via HVAC ducts: A new approach," in Proc. IEEE Global Telecommun. Conf., vol. 6, San Antonio, TX, Nov. 2001, pp. 3604-3607.

[9] P. Nikitin, D. D. Stancil, O. K. Tonguz, A. Cepni, A. Xhafa, and D. Brodtkorb, "Propagation model for the HVAC duct as a communication channel," IEEE Trans. Antennas Propag., vol. 51, pp. 945-951, May 2003

[10] O. K. Tonguz, D. D. Stancil, A. E. Xhafa, A. G. Cepni, P. V. Nikitin, and D. Brodtkorb, "An empirical path loss model for HVAC duct systems," in Proc. IEEE Global Telecommun. Conf., vol. 2, Taipei, Taiwan, Nov. 2002, pp. 1850-1854

[11] P. V. Nikitin, D. D. Stancil, O. K. Tonguz, A. E. Xhafa, A. G. Cepni, and D. Brodtkorb, "Impulse response of the HVAC duct as a communication channel," IEEE Trans. Commun., vol. 51, pp. 1736-1742, Oct. 2003
[12] O. K. Tonguz, A. E. Xhafa, D. D. Stancil, A. G. Cepni, P. V. Nikitin, and D. Brodtkorb, "A simple path loss model for HVAC duct systems," IEEE Trans. Veh. Technol., vol. 53, pp. 1203-1214, Jul. 2004

[13] P. V. Nikitin, D. D. Stancil, A. G. Cepni, A. E. Xhafa, O. K. Tonguz, and D. Brodtkorb, "Propagation modeling of complex HVAC networks using transfer matrix method [indoor communication application]," in Proc. IEEE Antennas, Propag. Soc. Int. Symp., vol. 2, Jun. 2003, pp. $126-129$.

[14] R. W. Chang, "Synthesis of bandlimited orthogonal signals for multichannel data transmission," Bell Syst. Tech. J., vol. 45, pp. 1775-1796, Dec. 1966.

[15] R. W. Chang and R. A. Gibby, "A theoretical study of performance of an orthogonal multiplexing data transmission scheme," IEEE Trans. Commun. Technol., vol. COM-16, pp. 529-540, Aug. 1968.

[16] S. B. Weinstein and P. M. Ebert, "Data transmission by frequency-division multiplexing using discrete Fourier transform," IEEE Trans. Commun. Technol., vol. COM-19, pp. 628-634, Oct. 1971.

[17] L. J. Cimini, Jr., "Analysis and simulation of a digital mobile channel using orthogonal frequency-division multiplexing," IEEE Trans. Commun., vol. COM-33, pp. 665-675, Jul. 1985.

[18] I. Kalet, "The multitone channel," IEEE Trans. Commun., vol. 37, pp 119-124, Feb. 1989.

[19] N. A. Zervos and I. Kalet, "Optimized decision feedback equalization versus optimized orthogonal frequency-division multiplexing for highspeed data transmission over the local cable network," in Proc. IEEE Int Conf. Commun., vol. 2, Boston, MA, 1989, pp. 1080-1085.

[20] E. Ayanoglu, V. K. Jones, G. G. Raleigh, J. Gardner, D. Gerlach, and K. Toussi, "VOFDM broadband wireless transmission and its advantages over single carrier modulation," in Proc. IEEE Int. Conf. Commun., vol. 6, Helsinki, Finland, 2001, pp. 1660-1664.

[21] J. G. Proakis, Digital Communications. New York: McGraw-Hill, 1983.

[22] T. M. Cover and J. A. Thomas, Elements of Information Theory. New York: Wiley, 1991.

[23] A. Xhafa, O. K. Tonguz, A. Cepni, D. D. Stancil, P. Nikitin, and D. Brodtkorb, "Theoretical limits of HVAC duct channel capacity for highspeed Internet access," in Proc. IEEE Int. Conf. Commun., vol. 2, New York, NY, May 2002, pp. 936-939.

[24] A. G. Cepni, A. E. Xhafa, P. V. Nikitin, D. D. Stancil, O. K. Tonguz, and D. Brodtkorb, "Multi-carrier signal transmission through HVAC ducts: Experimental results for channel capacity," in Proc. IEEE Veh. Technol. Conf., vol. 1, Vancouver, BC, Canada, Fall 2002, pp. 331-335.

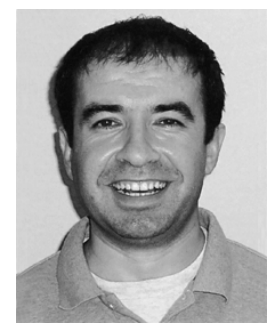

Ariton E. Xhafa (S'98-M'04) was born in Karbunare, Vlore, Albania. He received the B.S. degrees in electrical and electronics engineering and physics from Eastern Mediterranean University, North Cyprus, Turkey, in 1997, the M.S. degree in electrical engineering from the State University of New York at Buffalo, in 1999, and the Ph.D. degree in electrical and computer engineering from Carnegie Mellon University (CMU), Pittsburgh, PA, in 2003.

From 2000 to 2001, he was a Visiting Researcher at $\mathrm{CMU}$, doing research on design and performance analysis of communication systems. From 2002 to 2003, he was a Research Assistant in the Telecommunications Research Group, Center for Wireless and Broadband Networking, CMU, doing research on design and performance evaluation of wireless networks. From January 2004 to April 2004, he was a Research Associate at CMU, doing research on scheduling, handover management, and resource allocation in wireless networks. Currently, he is with the Communication Systems Laboratory, Texas Instruments Inc., Dallas, TX. His current research interests include design and evaluation of multiple-access control (MAC) protocols, handover management, and quality-of-service in wireless networks.

Dr. Xhafa has served on the Technical Program Committees of several IEEE conferences, including the VTC, ICC, and Globecom. He is a Member of Eta Kappa Nu, the IEEE Communications Society, the IEEE Vehicular Technology Society, and the IEEE Computer Society. 


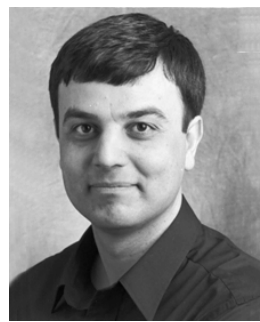

Ozan K. Tonguz (S'86-M'90) was born in Nicosia, Cyprus, in May 1960. He received the B.Sc. degree from the University of Essex, Colchester, U.K., in 1980, and the M.Sc. and Ph.D. degrees in electrical engineering from Rutgers University, New Brunswick, NJ, in 1986 and 1990, respectively.

$\mathrm{He}$ is currently a tenured Full Professor with the Department of Electrical and Computer Engineering, Carnegie Mellon University (CMU), Pittsburgh, PA. Before joining CMU in August 2000, he was with the Electrical and Computer Engineering Department, State University of New York at Buffalo (SUNY). He joined SUNY in 1990 as an Assistant Professor, where he was granted early tenure and promoted to Associate Professor in 1995, and to Full Professor in 1998. Prior to joining academia, he was with Bell Communications Research (Bellcore), Red Bank, NJ, between 1988-1990, doing research in optical networks and communication systems. He has published in the areas of optical networks, wireless communications and networks, high-speed networking, and security (in both wired and wireless networks). He is author or coauthor of more than 150 technical papers in IEEE journals and conference proceedings, and a book chapter in The Encyclopedia of Electrical and Electronics Engineers (New York: Wiley, 1999). He is the author of Ad Hoc Wireless Networks: A Communication-Theoretic Perspective (New York: Wiley, 2005). He was also the architect of the "High Performance Waveform (HPW)" that was implemented in Harris RF Communications' AN/PRC-117f multiband man-pack tactical radio. His industrial experience includes periods with Bell Communications Research, CTI, Inc., Harris RF Communications, Aria Wireless Systems, Clearwire Technologies, Nokia Networks, Asea Brown Boveri (ABB), and Intel. He currently serves as a consultant for several companies, law firms, and government agencies in the United States and Europe in the broad area of telecommunications and networking. He is also a Co-Director (Thrust Leader) of the Center for Wireless and Broadband Networking Research, CMU. More information about his research interests and his research group can be found at http://www.ece.cmu.edu/ tonguz/.

Dr. Tonguz, in addition to serving on the Technical Program Committees of several IEEE conferences (such as INFOCOM, GLOBECOM, ICC, and VTC) and symposia in the area of wireless communications and optical networks, currently serves or has served as an Associate Editor for the IEEE TRANSACTIONS on COMMUNICATIONS, IEEE Communications Magazine, and the JOURNAL OF LightwAVE TECHNOLOGY. He was a Guest Editor of the special issue of the JouRnal of LIGHTWAVE TECHNOLOGY and the IEEE Journal ON SELECTED AREAS IN COMMUNICATIONS on Multiwavelength Optical Networks and Technology, published in 1996.

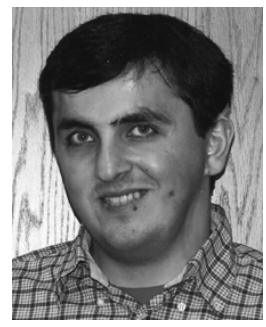

Ahmet G. Cepni (S’01) received the B.S. degrees in electrical and electronics engineering and physics from Bogazici University, Istanbul, Turkey, in 2000, and the M.S. degree in electrical and computer engineering from Carnegie Mellon University (CMU), Pittsburgh, PA, in 2002, where he is currently working toward the Ph.D. degree.

Since January 2001, he has been a Research Assistant with the Antenna and Radio Communications Research Group, CMU, and has been involved with indoor wireless communications via the HVAC duct project. His research interests include intelligent radio architectures, RF/microwave systems, and propagation models.

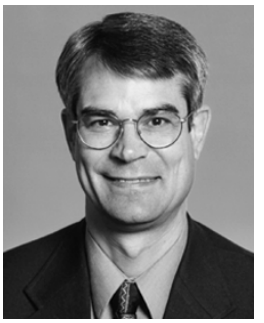

Daniel D. Stancil (S'75-M'81-SM'91-F'03) received the B.S. degree in electrical engineering from Tennessee Technological University, Cookeville, in 1976, and the S.M., E.E., and Ph.D. degrees from the Massachusetts Institute of Technology, Cambridge, in 1978,1979 , and 1981, respectively.

From 1981 to 1986, he was an Assistant Professor of Electrical and Computer Engineering with North Carolina State University, Raleigh. In 1986, he was an Associate Professor with Carnegie Mellon University (CMU), Pittsburgh, PA, where he currently is a Professor with the Department of Electrical and Computer Engineering, leads the Antenna and Radio Communications Group, and heads the newly established Center for Wireless and Broadband Networking.

Dr. Stancil was President of the IEEE Magnetics Society and was the Recipient of a 1985 Sigma Xi Research Award, presented by North Carolina State University. He was a Corecipient of a 1998 Science Award for Excellence, presented by the Pittsburgh Carnegie Science Center, a Research and Development 100 Award, and a Photonics Circle of Excellence Award for the development and commercialization of electrooptic technology. In 1996, he cofounded the Applied Electro-optics Corporation, Pittsburgh, PA, and was a leader in the development of the Virtual Laboratory, Electrical and Computer Engineering Department, CMU, which was a finalist for a 1996 Smithsonian Computerworld Award.

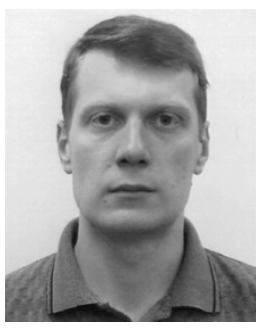

Pavel V. Nikitin (S'98-M'02) was born in Ashkhabad, U.S.S.R., in March, 1974. He received the B.S. degree in electrical engineering from Utah State University, Logan, in 1994, the B.S. degree in physics from Novosibirsk State University, Novosibirsk, Russia, in 1995, the M.S. degree in electrical engineering from Utah State University in 1998, and the Ph.D. degree in electrical and computer engineering from Carnegie Mellon University (CMU), Pittsburgh, PA, in 2002

He was a Research Associate with the Department of Electrical Engineering, University of Washington, Seattle, from October 2002 to April 2004, doing research on computer-aided design of mixed-technology systems. He is currently an Engineer with the Intermec Technologies Corporation, Everett, WA. His research interests include design, modeling, and simulation of antennas for RFID.

Dr. Nikitin is a member of Phi Kappa Phi and was a recipient of the 2000 Electrical and Computer Engineering Teaching Assistant of the Year Award, presented by CMU.

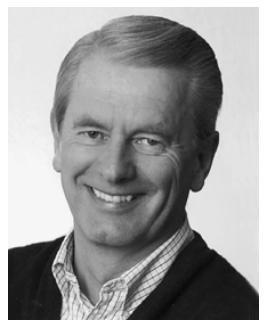

Dagfin Brodtkorb (M'81) was born in Norway in July, 1947. He received the M.S. and Ph.D. degrees in electrical engineering from the Norwegian Institute of Technology, Trondheim, Norway, in 1970 and in 1976, respectively.

From 1971 to 1980, he was a Research Engineer and Project Manager with the Norwegian Defense Research Establishment, Kjeller, Norway, where he was involved with radar and microwave systems. From 1977 to 1978, he was a Postdoctoral Researcher with the Lincoln Laboratory, Massachusetts Institute of Technology, Lexington, where he was involved with surface acoustic wave (SAW) technology. From 1980 to 1984, he was with A/S Informasjonskontroll, Asker, Norway, as a Manager of the Remote Sensing Group. From 1984 to 1995, he was Managing Director of MIROS A/S, Asker, Norway. Since 1995, he has been with the ABB Corporate Research Center, Billingstad, Norway, initially as a Senior Research Manager, then as a Technology Program Manager and as a Department Manager, and currently as Vice President (Research Director). He has authored several technical papers. 